\title{
Verbesserung des Menschen: Warum, und in welcher Hinsicht? Sechs Bücher zum Perfektionismus
}

Bernward Gesang: Die Perfektionierung des Menschen (Grundthemen Philosophie). Berlin 2007. Walter de Gruyter. 176 S.

Steven Lecce: Against Perfectionism. Defending Liberal Neutrality. University of Toronto Press 2008. 348 S.

Jonathan Marks: Perfection and Disharmony in the Thought of Jean-Jacques Rousseau. Cambridge University Press 2005. 191 S.

Michael Sandel: Plädoyer gegen die Perfektion. Ethik im Zeitalter der genetischen Technik. Mit einem Vorwort von Jürgen Habermas. Berlin University Press 2008. $175 \mathrm{~S}$.

Steven Wall: Liberalism, Perfectionism and Restraint. Cambridge University Press 2007 (zuerst 1998). 244 S.

Kimberly A. Yuracko: Perfectionism \& Contemporary Feminist Values, Bloomington 2003. Indiana University Press. 170 S.

\section{Einleitung}

Nach einem der zu besprechenden Werke verfolgt der Liberalismus eine minimalistische Ethik der Streitvermeidung unter den Einwohnern eines Landes (»seek peace« Marks 2005, 154). Um ihr Glück oder eine darüber hinausgehende Gerechtigkeit bekümmert er sich nicht. Genau dieser Liberalismus ist derzeit in der Defensive: Das Modell der europäischen Einigung, das sich von vereinigten Märkten auch vereinigte Staaten versprach, leidet durch geringe Wahlbeteiligung an Legitimationsdefiziten. In der Finanzkrise hat die Doktrin liberalisierter Märkte sogar auf ihrem eigensten Feld, der Wirtschaft, Schiffbruch erlitten. Für die politische Philosophie wäre das ein geeigneter Moment, Alternativen ins Spiel zu bringen - wenn sie denn welche anzubieten hätte. Es scheint nämlich, als habe die jahrzehntelange Dominanz des Marktradikalismus die projektive Phantasie (oder »utopische Energie«) der Philosophen ermüden lassen. Alternativen zum reinen Liberalismus wurden in letzten Jahren kaum noch ernsthaft diskutiert. Unter den wenigen Alternativen ist der Perfektionismus eine der interessanteren. Um Neuerscheinungen aus diesem Bereich soll es daher im Folgenden gehen.

Der Titel »Perfektionismus" ruft im deutschen Sprachraum vielfältige Assoziationen hervor: etwa die Manie, eine Sache besonders gut machen zu wollen, oder gnostische Denkmuster einer Vergöttlichung (henosis). Sie treffen allerdings nicht, was der angelsächsische Sprachraum damit meint, nämlich eine politische Ethik, die das langfristige Gelingen des menschlichen Lebens (kurz: »flourishing«) in den Mittelpunkt stellt. 
In Anlehnung an die aristotelische Redeweise der Vollkommenheit der Bürger geht es darum, Menschen in bestimmter Hinsicht »besser« zu machen. Aber Verbesserung schlechthin gibt es nicht, daher ist auf doppelte Weise zu differenzieren:

Wird die Verbesserung rein individuell vollzogen, kommt man kaum über die Thematik von Ratgebern hinaus ("Du musst dein Leben ändern«). ${ }^{1}$ Das ist noch nicht die Thematik des Perfektionismus. Wie das "machen« oben schon anzeigt, denkt dieser offen über Zugriffe von Institutionen auf die Entwicklung von Individuen nach. ${ }^{2}$ Darin manifestiert sich eine Spannung zum Liberalismus, der von Locke bis Rawls davon ausging, dass der Staat selbst keine "Konzeption des Guten« vertreten dürfe (was in der Praxis jedoch nie ganz stimmte). Erst eine institutionelle Praxis der Veränderung benötigt eine politische Ethik, die dem zeitgenössischen Liberalismus, der trotz politischer Rückschläge theoretisch noch dominiert, auf Augenhöhe begegnen kann. Dabei geht es in der Regel nicht darum, den Liberalismus zu sprengen, sondern ihn zu verändern, indem man ihn mit »Theorien des Guten« unterfüttert.

Beim Zugriff von Institutionen auf Individuen sind zudem zwei Praxen zu unterscheiden: Einerseits kann man Verbesserung "moralisch praktisch» (Kant, KU \ 88) verstehen - verbessert würde hier, traditionell aufklärerisch im Sinne der Milieutheorien, die Lebenssituation, und in der Folge der Charakter einer Person. Zwar kann sich eine Person letztlich nur selbst verbessern (oder "verwirklichen«, wie es hier oft heißt), doch Institutionen können günstige Rahmenbedingungen für eine günstige Entwicklung schaffen. Philosophische Überlegungen müssen dann sinnvolle Ziele und legitime Mittel einer solchen Politik der Selbstverwirklichungs-Ermöglichung erörtern. Zwei der zu besprechenden Werke nehmen zu dieser Thematik sehr grundsätzlich Stellung: Eines befürwortend, aber inhaltlich vage (Wall 2007), ein anderes ablehnend und bestimmt, dabei aber theoretisch unterkomplex (Lecce 2008). Zwei weitere und bescheidenere Werke beleuchten einerseits - und überraschenderweise - Rousseau als einen historischen Vorläufer dieses Denkens (Marks 2005) sowie die Bedeutung dieser Philosophie für den Feminismus (Yuracko 2003). Im Gegensatz zu den ersten Büchern sind diese kleineren Bücher inhaltlich außerordentlich ertragreich und stilistisch sehr ansprechend.

Anderseits kann man »technisch-praktisch» von einer biologischen Verbesserung der Menschen mithilfe modernster Technologien reden. Obwohl

1 Etwa Reinhold Ruthe: Die Perfektionismus-Falle und wie Sie ihr entgehen können, Moers 2009 .

${ }^{2}$ Dabei können auch Ratgeber als Techniken eines Regierungshandelns gedeutet werden (siehe Stefanie Duttweiler: Sein Glück machen. Arbeit am Glück als neoliberale Regierungstechnologie, Konstanz 2007). 
es weit von der aristotelischen Idee der Vervollkommnung entfernt scheint, ${ }^{3}$ wird gerade dies etwa im Rahmen des Transhumanismus als "Perfektionierung" verstanden. Die letzten beiden Werke nehmen dazu Stellung, erneut mit konträren Ergebnissen (Gesang 2007, Sandel 2008). Die normativen Folien dieser technischen Lesart von Perfektionierung setzen allerdings Positionen in der moralischen Lesart des Perfektionismus voraus. Diese zwei Debatten hält also nicht nur der Name zusammen.

\section{Zwei kleine Bücher zum Perfektionismus: Rousseau und die Frauen}

Beginnen wir mit den beiden »kleinen« und erfreulichen Büchern. Klein ist mit unter 200 Seiten nicht nur das Format, klein ist auch die Themenstellung. Gerade darin zeigt sich allerdings die philosophische Größe der Verfasser: Beide Werke haben handhabbare Themen, die eine originelle Fragestellung zielführend bearbeiten und am Ende mit so überraschenden wie überzeugenden Ergebnissen aufwarten. Das ist bei diesem Thema eher selten.

Das Buch der Juristin Kimberly A. Yuracko (2003) ist so klar geschrieben - man fühlt sich beim Lesen wie bei einem sonnig-klaren Gebirgsspaziergang -, dass es sich als "Einführung « in den Perfektionismus eignen würde, verfolgte es darüber hinaus nicht noch eine eigene Agenda, die ihr zugleich als Kritik der bisherigen Versionen der Theorie dient. Sie erläutert den Perfektionismus als diejenige politische Philosophie, die nicht nur wie der Liberalismus über formale Prozeduren, sondern auch darüber nachdenkt, "wie die Menschen leben sollten" (27) - nämlich so, dass sie "aufblühen«. Wenn Yuracko die perfektionistische Theorie des Guten "substantive» (27 u. ö.) nennt, ist das nicht als »substanzialistisch«, sondern vielmehr als "gehaltvoll" zu übersetzen (und so kann die zu erwartende Essentialismus-Schelte abgewiesen werden). Um welche Gehalte geht es?

Die feministische Liberalismuskritik von Yuracko geht - wie schon Karl Marx, der aber nicht genannt wird - davon aus, dass der Formalismus der liberalen Philosophie bestimmte Pathologien der modernen Welt nicht erfassen und folglich auch nicht kritisieren könne. Als Ausgangsbeobachtung dienen also Verhältnisse, die im Sinne des Liberalismus formal "gerecht« sind, da sie als freiwillig gelten, die aber dennoch kritisiert werden müssten und vom Feminismus auch kritisiert werden. Drei anschauliche Fallbeispiele ziehen sich durch das Buch: Prostitution ("sex-work»), Verdinglichung der eigenen Sexualität (»sexual objectification«) und das Leben als Hausfrau ("full time homemaker"). Nach Yuracko haben viele Menschen die Intuition, dass solche Lebensverhältnisse auch dann kritisiert werden können, wenn sie einmal selbst "gewählt« wurden. Eine solche Kritik ist aber in einem liberalen Denkrahmen schwer möglich. ${ }^{4}$ Dennoch berufen sich viele

3 Ein gesunder und starker Körper war jedoch schon für Aristoteles ein "Glücksgut«.

4 Schon Elizabeth Anderson ("What is the Point of Equality?», Ethics, Jan. 1999) hat 
feministische Kritiken auf den Liberalismus ${ }^{5}-$ zu Unrecht, meint Yuracko, weil der Liberalismus diese Kritik nicht begründen könne.

Nach ihrer Auffassung "funktionieren" die Kritiken nur, weil sie sich auf plausible, aber unausgesprochene Annahmen über das Blühen des menschlichen Lebens stützen. Yuracko will daher den unausgesprochenen perfektionistischen Subtext der feministischen Kritiken freilegen (»uncover«, 49), um ihn um so effektiver vertreten zu können. Ihr geht es also um Argumente in der politischen Auseinandersetzung, die möglichst überzeugend sein müssen. Die Zurücknahme zugunsten eines Neutralitätsprinzips erscheint als unnötige Schwächung der eigenen Position. An dieser Stelle ist ihr - etwa gegen Lecce (2008) - rechtzugeben: Neutralität ist Sache des Gesetzgebers, der zwischen verschiedenen Positionen vermittelt, nicht aber schon der Parteien, zwischen denen vermittelt werden soll. Hier geht es um "gute" Argumente, und es ist nicht zu sehen, warum man dabei auf perfektionistische Einsichten verzichten sollte. (Schliesslich benutzt ja auch die Gegenseite oft solche Argumente.)

An dieser Stelle macht Yuracko nun eine zweite Front auf. Kritisiert sie einerseits feministische Theorien, die sich auf den Perfektionismus stützen, ohne dies anzuerkennen, so bemängelt sie andererseits perfektionistische Theorien für ihre weitgehende Unbrauchbarkeit hinsichtlich der Beurteilung realer Verhältnisse. Die maßgeblichen Vertreter, so ihre scharfe, aber treffende Kritik, verbleiben in den Nebelzonen der Abstraktion und erfüllen daher ihren Zweck nicht. ${ }^{6}$ In der Tat bemühen sich diese Theorien, Konzeptionen des Guten als politisch förderungswürdig zu erweisen, ohne zu sagen, um welche Konzeptionen es dabei gehen soll oder gar anzugeben, an welche Art von Unterstützung dabei zu denken wäre. ${ }^{7}$ Einzig Martha Nussbaum (die ihren »Perfektionismus" nur selten als solchen ausflaggt) habe der Theorie die nötige Konkretion verliehen. Ihre Version sei aber für westliche Verhältnisse ebenfalls unbrauchbar, da sie im Kontext der Entwicklungspolitik Minimalstandards formuliere, die im Westen im wesentlichen schon (besser sollte man sagen: noch) erfüllt seien. Die femi-

auf die Absurdität des liberalen »Glücksegalitarismus« hingewiesen, welcher unverschuldete Ungleichheiten ausgleichen will (und daher z.B. Prämien auf Hässlichkeit auszahlen müsste), aber Ungleichheiten des Marktes unangetastet lässt, da sie im Prinzip auf freiwilligen Verträgen beruhen. Yuracko's Kritik setzt ähnlich an.

5 Behandelt werden u. a. Susan Okin, Margaret Jane Radin und Catharine A. MacKinnon.

6 Treffsicher behandelt sie Joseph Raz (The Morality of Freedom, Oxford 1986), Thomas Hurka (Perfectionism, Oxford 1993) und George Sher: Beyond Neutrality. Perfectionism and Politics, Cambridge 1997.

7 "The vagueness of abstract theory has ... kept perfectionism from having any real usefulness for individuals trying to determine how best to live their lifes and for societies trying to encourage individuals to live good lifes" (Yuracko, a.a.O., S. 40). 
nistische Kritik fokussiere im Westen auf andere Probleme, für die Nussbaum wenig zu bieten habe.

Wenn es Yuracko nun gelänge, in der feministischen Kritik perfektionistische Elemente aufzuweisen, hätte sie neben der methodischen Selbstkorrektur des $\mathrm{Fe}$ minismus zugleich einen wertvollen Beitrag zu einem gehaltvollen Perfektionismus geleistet. Wie werden diese beiden Stränge nun verbunden? Gehen wir dafür etwas näher in die Textur der Argumente. In den Versuchen, feministische Kritiken an den genannten Phänomenen »liberal« zu begründen, unterscheidet Yuracko vier Varianten. Alle versuchen aufzuzeigen, dass die vorgeblich freie Wahl nicht wirklich frei war, denn dann müsste auch ein Liberaler diese Verhältnisse ablehnen - aus formalen Gründen. Dies wird versucht, indem die Wahl als erzwungen bezeichnet wird (1); indem die vorangehende (verfehlte) Sozialisierung thematisiert wird (2); indem auf ungleiche Chancen für Frauen (3) oder eine Verletzbarkeit in der Zukunft verwiesen wird (4). Alle vier Varianten kann Yuracko zurückweisen.

Im Argument der erzwungenen Wahl (1) sei von "Zwang« nur im übertragenen Sinn die Rede. ${ }^{8}$ Gemeint seien eher unmoralische Angebote ("seductive offers"): Verführerische Angebote können Frauen dazu bringen, auf gewisse Weise über sich zu denken. Manche Dinge sollte man jedoch nicht zur Wahl stellen, denn schon eine solch korrumpierende Wahl habe einen schlechten Einfluss auf die Entwicklung von Personen. ${ }^{9}$ Hinter der liberalen Zwangskritik stehe also ein perfektionistisches Argument.

Das Argument der verfehlten Sozialisierung (2) lasse offen, warum manche Einflüsse der Sozialisierung abgelehnt würden und andere nicht. Man bemängele schlechte Wahlen, nicht die guten - die vorangehende Sozialisierung sei für die Kritik gar nicht das entscheidende Moment, das Kriterium sei vielmehr perfektionistisch. Ähnliches gelte für die "Authentizität« von Entscheidungen: Wann eine Wahl authentisch ist, vermag man kaum sicher zu sagen - gemeint sei damit vielmehr, dass es eine gute Wahl gewesen sei. Damit ist man ebenfalls wieder beim Perfektionismus gelandet.

Ähnlich verhält es sich mit dem Argument der ungleichen Chancen (3): Yuracko verweist auf Ungleichheiten, die von Feministinnen nicht kritisiert würden (etwa die verschiedenen Ligen von Männern und Frauen beim Baseball, 98). Angestrebt würde also nicht "neutrale» Gleichheit, sondern gleicher Zugang zu wertvollen Optionen - auch dies sei bereits perfektionistisch gedacht.

Das Argument der zukünftigen Verletzbarkeit schließlich (4) kranke daran, dass manche Entscheidungen im vollen Bewusstsein der Risiken gefällt würden - Feministinnen, die auf dieses Pferd setzten, wären in diesen Fällen gezwungen, auf ihre Kritik zu verzichten, was gleichwohl selten geschieht. Der Grund dafür sei wiederum, dass auch bei vollem Bewusstsein schlechte Entscheidungen gefällt werden könnten - schlecht seien sie aber, weil sie eine gute Entwicklung eher behindern als fördern (104).

${ }^{8}$ Ein Beispiel ist der "Tausch" einer unverdient guten Zensur gegen Sex: Die entsprechende Person erleidet im Falle der Verweigerung keine Gewalt; sie wird weder beraubt (sie bekommt sonst einfach die Zensur, die sie verdient hat) noch entrechtet (es gibt kein Recht auf eine Zensur über der eigenen Leistung) oder benachteiligt (zumindest, wenn nicht auch alle anderen dieses Angebot bekommen und annehmen). Dennoch würden die meisten sagen, dass mit diesem Angebot etwas nicht stimmt, die Frage ist nur: was?

9 Yuracko, a.a.O., S. 67. Gesang (2007) dagegen unterläuft dies. 
Der Perfektionismus leiste also das, was der Liberalismus nicht leisten kann: Er begründet die feministischen Einwände gegen Sexarbeit, Selbstvermarktung und Verhausfraulichung. Ein Beispiel von Methodenbewusstsein ist es nun, dass Yuracko das Gewonnene zuletzt als positive Theorie fasst. Was ist es, was ein gelingendes Leben - insbesondere von Frauen in der westlichen Welt - ausmacht und daher von Individuen und Gesellschaften angestrebt werden sollte? Wenn Yuracko an dieser Stelle (111ff.) nichtverdinglichte Sexualität, intellektuelle und moralische Entwicklung, Selbstliebe (im Deutschen würde man eher von Selbstachtung sprechen) sowie materielle Eigenständigkeit aufführt, so vermag man ihr kaum zu widersprechen. Man ist daher gespannt auf die liberale Antikritik (s.u. zu Lecce). Diese wird den Problematisierungsgrad dieses kleinen Meisterwerks allerdings nicht erreichen.

Das zweite kleinformatige Buch oszilliert ebenfalls um das normative Zentrum einer gelingenden menschlichen Entwicklung. Jonathan Marks (2005) räumt in seiner bemerkenswerten Rousseaustudie gleich mit mehreren überkommenen Deutungsschablonen auf. ${ }^{10}$ Ihm gelingt der Nachweis, dass Rousseau keineswegs der elliptische Extremist ist, für den die gängige Rezeption ihn hält (Marks rezipiert allerdings vorrangig angelsächsische Werke). Gemeinhin hält man Rousseau sowohl seine Verherrlichung eines vorsozialen Naturzustandes wie seine totale Auslieferung der Bürger an den Gemeinwillen vor. Beides sind denkbar radikale Positionen (völlige Gesellschaftslosigkeit hier, totale Vergesellschaftung dort), die sich zudem widersprechen. Als hermeneutisch sensibilisierter Leser rekonstruiert Marks einen anderen Rousseau, für den bereits der Naturzustand problematisch ist - damit verlagert sich die Ursache für die Zerrissenheit des Menschen von der Gesellschaft zurück in die menschliche Natur -, und der darum auch die radikale Trennung zwischen Natur und Gesellschaft nicht ernsthaft vertritt (37; »Original man is not original«, 25). Damit wird Rousseau zunächst als Theoretiker einer natürlichen Künstlichkeit des Menschen gewürdigt. ${ }^{11}$

Ist diese Umwertung am Rückblick orientiert, so birgt der Blick nach vorn eine weitere Überraschung: Marks' Relektüre legt eine Unterscheidung frei zwischen Aussagen zum Urzustand (in dem es auch problematische Züge des Menschen gibt) und zur "wahren Natur» des Menschen, die sich erst als Ziel einer gelingenden Erziehung einstellt. Marks liest

${ }^{10}$ Der Vergleich mit einer "rettenden Kritik» im Sinne Benjamins liegt nahe: Rousseau wird "recovered" (118).

${ }_{11}$ Das macht deutlich, dass Erkenntnisse, die zuweilen germanozentrisch Helmuth Plessner zugeschrieben werden, bereits Rousseau zugänglich waren. Marks' bezieht sich nicht auf Plessner, und das schadet nichts. 
Rousseau somit als Perfektionisten und Teleologen. ${ }^{12}$ Diese ungewöhnliche Deutung - immerhin ist die "perfectibilité» im Zweiten Discours, an dessen Wortlaut sich viele Deutungen festbeissen, eher negativ bewertet - gelingt ihm durch sein solides Handwerkzeug. Einerseits bedient er sich eines hermeneutischen Holismus: Marks schöpft seine Deutung aus einer Gesamtschau des Werkes. Vor allem, aber nicht nur im Emile, welches Rousseau als sein "größtes und bestes Buch« bezeichnete (Marks 2005, 4), findet er Belege für seine Thesen. Andererseits lässt er sich von der Cambridger »history of ideas" anregen, welche die rhetorischen Strategien philosophischer Texte aus ihrem politischen Umfeld neu deutet. ${ }^{13}$ Das führt Marks zur These, Rousseau habe manche seiner Anschauungen in seinen Texten gewissermaßen "versteckt" (111) und dabei auch Widersprüche in Kauf genommen, um eine größere politische Wirksamkeit zu erzielen. Aufmerksamkeit ist ihm ja zuteil geworden. Es ist eine plausible Annahme, darin eine Strategie zu vermuten - dass Kenntnisse der R hetorik heute weniger verbreitet sind als seinerzeit, spricht nur für die These, dass der Rezeption dies bislang entgangen ist.

Systematisch interessant ist, dass die philosophischen Großthesen Rousseaus sein Egalitarismus und sein Republikanismus - in dieser Rousseaudeutung als perfektionistisch fundiert erkennbar werden. Die normative Folie des Gesamtwerks ist eine Theorie des Glücks auf anthropologischer Basis: "Der Mensch" ist bei Marks' Rousseau keineswegs von Natur aus glücklich, sondern wird von widerstreitenden Antrieben geleitet. Zwischen diesen Kräften lässt sich allerdings eine Balance erreichen $(55,85)$, und dem dienen die ausgefeilte Erziehung und die politische Theorie. Diese glückliche Balance mache die "Perfektion" und das Glück des Menschen aus. Rousseaus Thema sei also »the natural perfection of a naturally disharmonious being " $(1,56)$. Das pointiert drei Thesen: Erstens ist der Naturzustand unharmonisch und daher kein sinnvolles Ziel der Politik - es gibt also keinen Widerspruch im Werk. ${ }^{14}$ Zweitens gibt es eine weitere Redeweise von Natur, die die Perfektion des Menschen bezeichnet. Drittens aber liegt diese Perfektion, wie Marks gegen Kantianische Deutungen hervorhebt, nicht "jenseits" der Natur (also auch nicht, wie im Transhumanismus, in ihrer Überwindung), sondern sie verbleibt "in" ihr und vermittelt nur zwischen den Gegensätzen.

$12 »[\mathrm{H}]$ uman nature consists in an end or perfection ... Rousseau's understandig of nature is teleological" (38). Ähnlich Rainer Bolle: Jean Jacques Rousseau. Das Prinzip der Vervollkommnung des Menschen durch Erziehung und die Frage nach dem Zusammenhang von Freiheit, Glück und Identität, Münster 2005.

13 Siehe etwa Quentin Skinner: Reason and Rhetoric in the Philosophy of Hobbes, Cambridge 1996.

${ }_{14}$ Wie Marks zeigt, wird Rousseau emphatisch, wenn er Zwischenstufen der Zivilisation - die goldene Mitte - beschreibt: etwa das "primitive Stadium« (im Unterschied zum Urzustand) oder Schweizer Kommunen. 
Es erscheint nach dieser Reinterpretation als denkbar, Rousseau in die Tradition des "politischen Aristolismus" einzureihen, ${ }^{15}$ denn dieser Rousseau kommt Aristoteles in einigen Kernpunkte nahe (mit der Teleologie, der Glücks- und der Mesoteslehre und natürlich in der republikanischen Staatstheorie). Diese Auseinandersetzung führt Marks nicht mehr. Stattdessen setzt er sich zum Schluss mit einem Neoaristoteliker auseinander: Charles Taylor, der sich in wichtigen Werken immer wieder auf Rousseau bezog, wird unbarmherzig nachgewiesen, dass sich seine hermeneutisch wenig sensible Rousseauinterpretation nicht halten lässt. Mehr noch, Marks scheint es geärgert zu haben, dass Taylor Anschuldigungen gegen Rousseau erhebt (bzw. wiederholt), die nach Marks viel eher auf seine eigene Theorie zutreffen.

Rousseau habe, so Marks, gerade durch seinen Bezug auf die Natur ein Kriterium dafür, wann eine Lebensform »authentisch"sei und wann nicht - nämlich dann, wenn sie der Natur der betreffenden Person entspreche (die sich z.B. in seinem Leib verkörpere, 122). Dieses Kriterium erlaubt es Rousseau sogar, dem Zugriff der Gesellschaft auf das Individuum Grenzen zu setzen - Rousseau sei zwei kein Liberaler, aber im Gegensatz zu Taylor kenne er Schutzzonen für das Individuum (148). Diese fehlen Taylor deswegen, weil er als Quelle für eine authentische Identität nur die Gemeinschaft zulässt. Man kann sich in diesem Denkrahmen schwerlich von der Gemeinschaft zurückziehen, ohne sogleich seine Identität zu gefährden. ${ }^{16}$

Rousseau stellt also nicht nur eine Herausforderung für den Liberalismus dar. Einerseits ist er auch für die Kommunitaristen ein Stachel im Fleisch, da seine durchschlagende Liberalismuskritik den Kommunitarismus als verwässerte und nicht völlig durchdachte Position erscheinen lässt. Andererseits ist Rousseau - wie Marks abschließend bemerkt (154) - bei aller Kritik anschlussfähig an die liberale politische Philosophie. Systematisch heißt das, dass ein liberaler Perfektionismus möglich ist. "Liberal« meint hier allerdings, Schutzräume für Individuen zu schaffen, nicht aber, die Gemeinschaft kritiklos dem Marktmechanismus auszuliefern, denn gerade diesen (bzw. den Typus des »bourgeois«) trifft Rousseaus härteste Kritik (151). Die Verträglichkeit von Perfektionismus und Liberalismus bleibt also eine harte Nuss. Zwei weitere Werke befassen sich mit dieser Thematik auf einem höherem Abstraktionsgrad.

15 Christoph Horn/Ada Neschre-Hentschne (Hg.): Politischer Aristotelismus. Die Rezeption der aristotelischen "Politik" von der Antike bis zum 19. Jahrhundert, Stuttgart 2008.

16 Dasselbe spielt Marks für die Anerkennung durch - diese kann auch von der falschen Seite kommen, Taylor fehle aber das Kriterium, dies zu unterscheiden. Leider geht Marks nur auf Taylors Werke bis 1995 ein. Eine kritische Funktion der »ersten Natur« gibt es später auch bei Karl Marx; siehe Christoph Henning: "Karl Marx", in: Eike Bohlken/ Christan Thies, Handbuch Anthropologie, Stuttgart 2009 (im Druck). 


\section{Zwei ehrgeizige Bücher zum Perfektionismus: Die Frage der Neutralität des Staates}

Was könnte ein klassisch liberaler Philosoph (im Sinne von Locke, Hayek und Rawls) den bisherigen Argumenten antworten? Eine mögliche Antwort wäre, dass Yuracko und Rousseau ihre politische Ethik aus einer »Konzeption des Guten« ziehen (nach Rawls also eine »comprehensive theory« vertreten): Yuracko macht sich für »substantielle« Aussagen über Möglichkeiten gelingenden Lebens stark, Marks verteidigt einen politischen Philosophen, dessen Hauptthema die "Perfektionierung « eines von Natur aus unharmonischen Wesens sei. Der Liberalismus als politische Theorie steht jedoch vor dem Problem, dass es nicht nur eine Konzeption des Guten gibt. Wie aber können in einem Gemeinwesen verschiedene Konzeptionen bestehen, ohne sich wechselseitig einzuschränken? Diese Frage scheinen Yuracko und Marks nicht zu stellen. Das Hauptargument des Buches von Steven Lecce (2008) ist daher zunächst triftig: Diese Frage könne nicht auf "epistemische» Weise gelöst werden. "Wahrheit" sei eine Selbstbeschreibung, die den Konzeptionen des Guten verhaftet bleibe und keinen übergreifenden Konsens stiften könne. Mit Locke gesagt: Jede Religion ist sich selbst die Orthodoxie. ${ }^{17}$

Die Stärke dieser - wenngleich nicht originellen ${ }^{18}-$ Kritik von Lecce zeigt sich daran, dass die abstrakte Verteidigung des Perfektionismus bei Steven Wall (2007) keine Antwort darauf hat. Wall vertritt die Auffassung, dass eine Gruppe mit der "richtigen" Konzeption (vorausgesetzt, sie hat auch die »richtigen" Gründe) berechtigt sei, ihre Prinzipien einer anderen Gruppe aufzuzwingen. ${ }^{19}$ Die staatliche Neutralität gegenüber Konzeptionen des Guten ist damit außer Kraft gesetzt. Genau solche Politiken möchte Lecce ausschließen, da sie mit dem liberalen Toleranzprinzip unverträglich sind. Bezogen auf die Ausgangsdiskussion lässt sich ermitteln, was das praktisch meint: ${ }^{20}$ Wall hält es für falsch, prozeduralen Argumenten (über das Gerechte) immer einen Vorrang vor inhaltlichen Argumenten (über das Gute) einzuräumen (91). Es sei denkbar, einer Gruppe »richtige« Prinzipien aufzuzwingen, obwohl Liberale das für ungerecht halten. Als Beispiele für solche Prinzipien nennt Wall den Zwang zur vegetarischen Ernährung, ein Verbot der Abtreibung und die Bestrafung der Beihilfe zum Selbstmord

17 "[T]he distinction between syour religion and strue religion is entirely without force $($ Lecce 2008, 25).

${ }_{18}$ Die Einsicht ist nicht neu - Lecce entnimmt sie Locke und Rawls (den er allerdings auch kritisiert), erinnert sei darüber hinaus an Hayek oder Karl Mannheim.

${ }_{19}$ Wall 2007, 82. "We should not give weight to harms which are only harms because people have false beliefs« (89; vgl. 86: »they are wrong«). Diese Position schreibt Wall Mill zu, aber mit Einschränkungen stimmt er ihr zu und schließt: "This refutes the democratic idea of toleration« (91).

${ }^{20}$ Die Texte von Wall und Lecce sind wegen der abstrakten Themenstellung, aber auch weil ihnen im Vergleich zu Marks und Yuracko die klare Darstellungsweise fehlt, schwerer durchdringbar: Gedanken erscheinen in assoziativer Folge; ein Rückbezug auf Ausgangsüberlegungen fehlt zuweilen. Der Leser muss Arbeit leisten, die Sache der Autoren gewesen wäre. Walls Position ergibt sich erst, wenn man Textstellen zusammenfügt. 
(75 Fn.). Wie kommt Wall zu diesem Schluss? Man müsse, so Wall, alle Seiten betrachten: Zwar geschehe der gezwungenen Gruppe möglicherweise »symbolisches Unrecht, doch wenn die zwingende Gruppe auf die Durchsetzung ihrer Prinzipien verzichte, geschehe (so seine "content consideration", 91) reales $\mathrm{Un}$ recht, und das wiege schwerer. ${ }^{21}$ Wall gesteht also einer Gruppe zu, über die richtigen Prinzipien zu verfügen, andere Gruppen haben entsprechend die falschen und dürfen "gezwungen" werden. Offensichtlich verfehlt Wall damit die Problematisierung von Lecce: Es ist ja gerade strittig, welches die richtigen Prinzipien sind. Gibt es einen Wertepluralismus, wird jede Seite ihre Auffassung als die richtige hinstellen. Der Satz "meine Auffassung ist die wahre entstammt dem "geistigen Tierreich" (Hegel) und fügt dem Streit nichts hinzu. Darum befindet Lecce, dass Perfektionisten wie Joseph Raz das Problem des Pluralismus verfehlen. ${ }^{22} \mathrm{Ob}$ das für alle Perfektionisten gilt, ist fraglich; auf Steven Walls Version (die in der Raz'schen Linie liegt) dürfte dieser Einwand jedenfalls zutreffen.

Wie geht nun Lecce selbst mit diesem Problem um? Immerhin könnten Perfektionisten einwenden, dass der epistemische Zweifel sich auch auf die Prozeduren erstrecken müsste. Der Liberale hätte dann wenig gewonnen. Wenn zwischen konkurrierenden Konzeptionen des Guten keine Einigkeit herzustellen ist, woher soll dann Einigkeit über gerechte Prozeduren kommen? ${ }^{23}$ Lecces Lösung besteht in der Unterscheidung zwischen epistemischer und moralischer Vernünftigkeit (»reasonableness", 169), die bei Rawls nicht geschieden seien. Selbst wenn auf epistemischer Ebene kein Konsens zu erzielen sei, lasse sich auf moralischer Ebene Einigkeit über Verfahren herstellen, solange allen Parteien eine demokratische Gleichheit zugestanden werde (193). ${ }^{24}$ Lecce glaubt nun, der Perfektionismus müsse an diesem Kriterium scheitern - und in der Tat ist sein Kronzeuge Raz nicht nur Perfektionist, sondern auch Anti-(oder Non-)Egalitarist. Ohne dass wir die beiden Bücher bisher näher vorgestellt haben, wissen wir damit bereits, dass sie sich widersprechen. Beschreiben wir aber noch deren Gehalte etwas näher:

Walls Buch möchte überprüfen, ob der Perfektionismus gut begründet (a) und ob er mit dem Liberalismus verträglich ist (b). Den Perfektionismus im Sinne von Raz versucht er zu stärken, indem er theoretische Bedenken an John Rawls' Neutralitätsprinzip anmeldet (a). So könne der Staat in Situationen, in denen sich Bürger gegenseitig verfolgen, nur entweder eingreifen und die Angegriffenen schützen, oder dies nicht tun und

21 "If they do not enforce these views, injustice will result" (Wall 2006, 86; cf. 90).

22 "Raz's argument does not adress this [rival truth-claims, CH] at all" (Lecce 2008, 127). Raz's Unterstellung, der Perfektionismus sei "authoritive only when reasonable« (124) umgehe das Problem schlichtweg.

${ }^{23}$ Lecce 2008, 164 nennt das die "reflexivity thesis" (cf. Wall 2007, 104). Andere wenden es positiv: Wenn wir vom Einen wissen können, warum nicht vom Anderen? (Raz, 126f.; Hurka, 163; Sher, 144 f.; s. o., Fn. 5).

${ }^{24}$ Seltsamerweise nur bezweifelt Lecce einen Konsens auch hinsichtlich moralischer Fragen (179). 
einen Schaden zulassen. Doch keine dieser Handlungen sei "neutral" (37). ${ }^{25}$ Das ist möglich, spricht aber noch nicht speziell für den Perfektionismus.

Auf den Liberalismus hingegen (b) kommt Wall nicht mehr zurück. Wie die genannten Beispiele schon andeuteten, vertritt Wall eher konservative als liberale Positionen: Der Staat soll im Prinzip einige Lebensformen vorziehen, und konkrete Beispiele für eine solche Politik weisen ebenfalls in die konservative Richtung. ${ }^{26}$ Das liegt vermutlich daran, dass nicht die liberale Autonomie im Vordergrund steht, sondern - wie schon bei Raz - die »Werte«. Die Autonomie (verstanden als das Ideal, selbst etwas "aus sich zu machen «) ${ }^{27}$ ist nur deswegen förderungswürdig, weil sie Teil einer wertvollen Kultur ist, nämlich der westlichen $(121,175)$. Sie darf daher eingeschränkt werden (185), wenn ein Wert auf dem Spiel steht. Antworten auf die Frage, woher wir von diesen Werten wissen und warum sie für alle Bürger verbindlich sein sollen, sucht man allerdings vergebens. Daher ist auch nicht klar, warum nun gerade konservative Werte - und nicht etwa egalitäre - bevorzugt werden.

Für Wall stellt die »Wahrheit«, auf die er die Politik verpflichten möchte, kein Problem dar, ${ }^{28}$ doch wüsste man schon gern, woher er diese beziehen will. Am Ende steht ein Plädoyer für eine Politik, die (in westlichen Gesellschaften) Autonomie nicht nur respektieren, sondern auch stärken soll (206), die sich aber auch für spezielle Werte (etwa in der Kunst, 213) einsetzen soll. Wie bereits Yuracko bemängelt hat, bleiben dabei Zwecke und Mittel, oder Werte und Wege, reichlich vage. Es bleibt daher letztlich offen, wie Wall es mit dem Liberalismus hält und warum gelten soll: "ultimately it is the flourishing of human beings that matters" (182).

Lecces Buch hingegen möchte aus klassisch liberaler Sicht zeigen, dass der Perfektionismus auf dem Holzweg ist. Der Perfektionismus hat bei ihm von vornherein keine Chance, da Lecce nur seine schlechten Seiten wahrnimmt. Diese kritisiert er zwar mit Recht, aber seine Schlussfolgerungen stehen damit auf dünnen Beinen - was ihn nicht hindert, seine Punkte ausgiebig zu wiederholen. Schon der erste, erfreulicherweise ideengeschichtlich orientierte Teil startet mit einem Reigen dreier offen-

${ }^{25}$ Die Neutralitätsthese von Rawls (der Staat dürfe selbst keine Konzeption des Guten vertreten) wird aufgegliedert in Thesen über Toleranz und öffentliche Rechtfertigung, an denen Wall jeweils Unklarheiten moniert.

26 "[P] olitical authorities should actively favor some ways of life over others" (Wall 2007, 207). Inhaltlich macht sich Wall neben dem Verbot der Abtreibung etwa für eine restriktive Immigrationspolitik stark (228).

27 Autonomie versteht Wall als »the ideal of people charting their own course through life ... and making something out of their lifes according to their own understanding of what is valuable» (128).

28 "We want (and should want) our political life to be based on true doctrines about the world« (Wall 2007, 178). 
sichtlich illiberaler Autoren aus der Britischen Tradition, deren "Widerlegung « daher keine große Schwierigkeit ist - Lecces Argumente treffen darum jedoch noch nicht den Perfektionismus, sondern nur die Illiberalität dieser Autoren. ${ }^{29}$

Der zweite Teil setzt diese Strategie fort: Zwar sind Lecce einschlägige Autoren wie Hurka oder Sher (Fn. 5) bekannt, direkt setzt er sich jedoch nur mit einem der angreifbarsten $(\mathrm{Raz})^{30}$ und einem eher entlegenen Vertreter (Dworkin) des Perfektionismus auseinander.

An Raz kritisiert Lecce das Fehlen liberaler Elemente: Nicht die Rechte Anderer, sondern das eigene Wohlbefinden lege bei Raz den Rahmen der für die personale Autonomie konstitutiven wertvollen Aktivitäten fest; in letzter Instanz entscheidet zudem nicht das Individuum, sondern der Staat, welches Set von Optionen wertvoll sei (Lecce, 102). Zudem kann Lecce sein Standardargument anbringen: Raz harmonisiere die Konflikte innerhalb der bürgerlichen Gesellschaft und lasse einen echten Widerstreit der Auffassungen nicht zu - dieser sei aber die Regel, und daher sei Raz' Theorie inadäquat (131).

Dworkins moderate Perfektionismus-Adaption sieht ein gutes Leben als ein herausforderndes Leben an (»challenge «) ${ }^{31}$ und schlussfolgert, dass allen Bürgern gleiche Chancen auf ein solches Leben zustehen (Lecce, 147). Lecce kritisiert daran im Rückgriff auf ältere Kritiken (etwa von Richard Arneson), dass sich weder Dworkins Egalitarismus noch seine Orientierung am Guten mit der »liberalen Neutralität« vertrage. Es fehlten ihm guten Argumente gegen den Paternalismus (150).

Der dritte Teil beschreibt Lecces eigene Ansicht: Eine liberale Neutralität des Staates könne auf perfektionistischer Grundlage nicht erreicht werden. Die moralische oder »demokratische Gleichheit« (im Sinne von Elizabeth Anderson, 183) verlange, dass jeder Bürger gleiche Rechte habe, unabhängig davon, wie ein anderer oder wie der Staat ihn einschätze. Die einzig adäquate politische Theorie sei daher der Kontraktualismus, da in dessen Abstraktionsgrad die (fiktiven) Staatsbürger "gleich"seien und nicht benachteiligt werden könnten. ${ }^{32}$ Was aber hat Lecce damit geleistet? Er hat uns seine eigene Überzeugung in Modellform dargelegt. Doch hat er weder begründet, warum der "Wert« der liberalen Neutralität alle anderen Werte schlägt, noch hat er dargelegt, wie man durch bloße Abstraktion bei

29 Erörtert wird die Kritik von Jonas Proast an Locke, von James F. Stephen an J. S. Mill, und von Patrick Devlin an H.L.A. Hart. Die Antikritik von Lecce läuft stets darauf hinaus, dass die Autoren für ihre Position eine "Wahrheit« beanspruchen (die "wahre Moral" dürfe erzwungen werden), die nicht zur Verfügung steht.

30 Vgl. auch Yuracko, 28 ff. Raz' Buch widerspricht sich schon in der Anlage: Gilt personale Autonomie eingangs als Eigenwert, gilt sie im weiteren Verlauf nur als wertvoll, wenn sie die "richtigen« Werte wählt (aber welche sind dies?). Angreifbar ist zudem die kollektivistische und anti-egalitäre Ausrichtung.

31 Ronald Dworkin: The Foundations of Liberal Equality, Tanner Lectures, Salt Lake City 1990.

32 Lecce (201ff.) setzt sich daher für Rawls' ältere Theory of Justice und gegen deren spätere Modifikatonen ein. 
einem echten Widerstreit der Werte zu einer politischen Selbstregierung kommen soll. Vor allem aber hat Lecce keinen Stich gegen einen intelligenteren Perfektionismus erzielt: Lecce traktiert ihn mit einer abstrakten "Wahrheit«, die im idealen Modell immer recht hat - und etwas anderes bekommt sein Kontraktualismus nicht in den Blick. Die Stärke des Perfektionismus liegt jedoch woanders.

Nehmen wir die Ausführungen Yurackos ernst, ist der Perfektionismus am stärksten in der nicht-idealen Wirklichkeit, da er reale Anliegen der Menschen auf eine Weise artikuliert, die abstrakten liberalen Prinzipien unzugänglich ist. Eine solche philosophische Theorie muss auch soziale Kontexte mit reflektieren, und daran fehlt es bei Lecce. Wall stellt zurecht heraus, dass das Thema des Perfektionismus nicht allein die (negative) Sicherung von Freiheitsrechten ist, sondern vielmehr die (positive) Schaffung und Förderung eines autonomen und gelingenden Lebens ("respecting" vs. "promoting autonomy", 206). Gegen solche Anliegen ist der Prinzipialismus von Lecce eine stumpfe Waffe. Ein Modell, in dem die idealen Voraussetzungen einer demokratischen Selbstregierung immer schon gegeben sind, sagt wenig gegen eine Theorie aus, die über die Schaffung und Erhaltung eben dieser Voraussetzungen (eine "Staatsbürgerqualifikationspolitik", wie Ulrich K. Preuss es einmal nannte) sowie über darüber hinausgehende Gelingensbedingungen des menschlichen Lebens nachdenkt.

Der Perfektionismus wird relevant, wenn solche Voraussetzungen fehlen oder erodieren. So feierte der Perfektionismus sein politisches Comeback, als konservative Autoren wie Lawrence Mead moralisch desaströse Auswirkungen des Wohlfahrtsstaates behaupteten (und New Labour auf den Zug aufsprang). Seither bedienen sich westliche Staaten einer paternalistischen "Aktivierungspolitik« gegenüber Arbeitslosen. Ein weniger abschreckendes Beispiel ist John Stuart Mills Liberalismuskritik: Wenn der Liberalismus seine Voraussetzungen nicht für alle Menschen sicherstellt, kann er hochgradig exkludierend werden (etwa gegenüber Frauen und Arbeitern - Mill sah nicht nur eine Erweiterung des Wahlrechts, sondern auch der Erziehung vor). Heute wird der Perfektionismus etwa, obzwar nicht immer unter diesem Titel, in der Rückkehr der Philosophie des Glücks diskutiert: Der jahrzehntelange Marktradikalismus hat nicht nur alle ungleicher und viele ärmer gemacht, sondern auch ein Sinnvakuum hinterlassen. ${ }^{33}$ Und schließlich ist Perfektionismus gar nicht so selten: In Erziehung und Ausbildung geht es täglich darum, Fähigkeiten möglichst weitgehend zu entwickeln. ${ }^{34}$ Es ist also ein berechtigtes Anliegen, neu über politische Bedingungen des "guten Lebens" nachzudenken.

Angesichts der Aktualität des Themas lassen Wall und Lecce die Leser etwas enttäuscht zurück: Ihre Bücher sind nicht nur weniger klar formuliert und (wahrscheinlich darum) länger als die von Marks und Yuracko, sie gehen auch seltsam dogmatisch vor. Zwar behandeln sie eine Anzahl von Argumenten anderer Autoren, aber diesen wird meist nur angekrei-

33 Siehe z. B. Robert E. Lane: The Loss of Happiness in Market Democracies, New Haven 2000, sowie Günter Dux: Wozu denn Gerechtigkeit, Weilerswist 2008 (und meine Besprechung auf Literaturkritik.de, 4/2009).

34 Emile, auf dem Marks aufbaut, ist ein Erziehungsbuch. Der Bolognaprozess teilt dieses Ziel leider kaum noch. 
det, dass sie dem von ihnen selbst vertretenen Punkt nicht gerecht werden. Diese Übung bleibt müßig, solange es an starken Argumenten für die eigene Position fehlt. Da Wall und Lecce auf unterschiedlichen Ebenen diskutieren (deren Gemeinsamkeit nur der hohe Abstraktionsgrad ist), ist am Ende nicht einmal klar, auf welcher Grundlage eine Entscheidung möglich wäre. Man kann nur den Dissens konstatieren und müsste weitere Argumente anfordern. ${ }^{35}$ Ein Blick auf andere Werke zum Thema lässt vermuten, dass eine Entscheidungsgrundlage am ehesten von der Anthropologie in Verbindung mit einer empirisch informierten Sozialtheorie kommen könnte. ${ }^{36}$ Damit sind wir bei den letzten Büchern angekommen - denn Michael Sandel macht dies am Beispiel der Bewertung einer ganz anderen Perfektionierung beispielhaft vor.

\section{Zwei Bücher zur Anthropotechnik}

Das Beunruhigende an den Möglichkeiten der Biotechnologie ist, dass das soeben beschworene anthropologische Fundament schwankend wird, wenn der Mensch beginnt, sich selbst umzubauen. »Der Mensch« ist kein Maßstab für die Frage, was er mit sich selbst anstellen darf. Doch eben diese Frage stellen sich die Bücher von Michael Sandel und Bernward Gesang: Wie sind die Möglichkeiten des "human enhancement" ethisch zu bewerten? Wenn auch sie unterschiedliche Schlussfolgerungen ziehen, liegt dies also nicht nur an ihren verschiedenen Anthropologien. Die Grenzen der Möglichkeiten des Menschen, die im moralischen Perfektionismus ein Argument sind (etwa wenn die sozialpolitische "Aktivierung« von Betroffenen unmögliche Dinge abverlangt), sollen ja gerade überwunden werden. Der Dissens geht daher auch auf unterschiedliche Sozialtheorien zurück, in die die Untersuchungen eingebettet sind.

Zunächst eine Begriffsklärung: Die Bücher sind keine allgemeinen Abhandlungen zur Gentechnik, denn diese kann ja auch medizinisch, d.h. zur Heilung von Krankheiten genutzt werden. Als Perfektionierung des Menschen (»human enhancement") wird der Sonderfall einer "technischen Verbesserung normaler Eigenschaften des gesunden Menschen durch Eingriffe in dessen Körper «erstanden (Gesang, 4). Mit einigen Einschränkungen begrüßt Gesang solche Möglichkeiten, während Sandel sie weitestgehend ablehnt. Das Erstaunliche ist allerdings,

35 »[E] in trockenes Versichern gilt aber gerade soviel als ein anderes" (G. W. F. HegeL, Phänomenologie, Frankfurt/M. 1970, 71).

36 Der Theorienpluralismus ist in diesem Disziplinen durch die Nähe zur Empirie besser beherrschbar als in politischen Werturteilen. Denken Yuracko und Wall in Ansätzen sozialtheoretisch, so argumentieren Hurka und Sher (wie Marks' Rousseau) eher anthropologisch. Lecce dagegen setzt an die Stelle des "Menschen" ein Idealbild des Bürgers ("hypothetical contractors", 163, vgl. 217), aus dem er weiteres ableitet. Das ruft die Kritik des jungen Marx an der nur "politischen" Emanzipation wach ("Zur Judenfrage", MEW 1, 354 ff.). 
dass beide ihre Überlegungen nicht auf Abgrenzungen der technischen Eigenarten der Eingriffe aufbauen - die Bücher sind also wenig technisch (Gesang zählt zwar Techniken auf, doch diese Details spielen für seine Argumentation gar keine Rolle). Beide argumentieren vielmehr aus einem Kontinuum von harmlosen Veränderungen (etwa einer engagierten Erziehung) bis zu »harten" Eingriffen wie einer Züchtung von Super-Athleten, allerdings mit disparaten Schlussfolgerungen. Gesang zieht aus bereits geübten Praxen der Verbesserung (64) die Lizenz zu einer (wenn auch nicht grenzenlosen) Erweiterung dieser Praxen; Sandel nimmt die beängstigenden Aussichten zum Anlass, auch bereits geübte Praxen ethisch zu hinterfragen.

Beginnen wir mit Bernward Gesangs Befürwortung. Sie stützt sich auf einen stark dekontextualisierten Utilitarismus. Dies erklärt bereits zum Großteil seine Position: Vieles, was sinnvollerweise gegen eine Ausweitung des "enhancement" vorgebracht werden könnte (und was sich etwa bei Sandel findet), kommt in dieser abstrakten Ethik nicht vor. Eine der seltenen Ausnahmen sind "ästhetische Interessen« der Mehrheit der Menschen (137). ${ }^{37}$ Allerdings scheint diese Verkürzung von Einwänden der Bevölkerung auf "ästhetische« den ethischen Gehalt solcher Einwände gerade nicht ernst zu nehmen. Tatsächlich steht Gesangs Ethik vor einem Dilemma, wenn er den Utilitarismus durch eine Berücksichtigung der tatsächlichen Wünsche der Menschen »humanisiert» (44): Sie ist zerrissen zwischen einer Bestätigung der ohnehin herrschenden Meinung (die sich allerdings schnell ändern kann), ${ }^{38}$ und einer technoiden Attitüde der "Aufklärung« eben dieser Meinungen, die stark kontraintuitive Werturteile fällt. ${ }^{39}$

Der Schwerpunkt des Buches liegt auf dem zweiten Element: Es geht nicht um eine Demokratisierung der Entscheidungspraxen, wie man sie aus Lecces Standpunkt vielleicht erwarten könnte, sondern um eine schön paradoxe - objektive Beurteilung des vermutlichen subjektiven Glückszuwachses durch solche Praxen. Der Gestus des Buches ist der eines wohlwollenden Gesetzgebers, ${ }^{40}$ der über das nötige Wissen verfügt und

37 Gesang, 137. Diese Interessen führen Gesang zu einem »Verbot« von Eingriffen, die den »Körper als menschlichen Körper unkenntlich machen« (156). Das verbietet aber nur extremste Eingriffe (»Chimären«).

38 „Betrachtet man diese Interessen mit, nähert sich das utilitaristische Urteilen automatisch [?] den moralischen Intuitionen der Mehrheit der Bevölkerung an« (44). Gesang meint hier die "externen" Interessen Dritter. Nimmt man die Interessen der Betroffenen hinzu (die die Autorität der ersten Person haben, welches Glück sie wünschen), so bleibt wenig übrig, was Gesang dem jeweiligen status quo ethisch entgegensetzen könnte.

39 Es gehe darum, »Interessen durch Argumente aufzuklären« (137). Doch Argumente wie die, dass Natur keinen Eigenwert habe, sind den Intuitionen vieler Menschen schroff entgegengesetzt. Gesang kann hierfür nicht den naturalistischen Fehlschluss bemühen (cf. 112), da er selbst einen solchen begeht, wenn er schließt, die Natur habe keinen Eigenwert, weil die Menschen sie bisher so stark zerstört haben (119).

${ }^{40}$ Das Buch endet mit einem Katalog von Erlaubnissen, Verboten und "Staatliche[n] Pflicht[en]» (156). 
nur aus pragmatischen Gründen auf Erkenntnislücken der Bevölkerung Rücksicht nimmt. Was ist nun dieses Wissen, das Gesang in dieser Angelegenheit reklamiert? Gesangs Methode besteht aus dem Gedankenspiel, von möglichen Risiken und Nebenwirkungen solcher Technologien abzusehen (der "Idealfall«, 36, 143) und zu überlegen, ob das Ergebnis das individuelle "Glück" der Menschen maximieren würde (nicht: ob wir als Gesellschaft das wollen können). Der Reichweite der Eingriffe wird dann je nach zu erwartenden Glückseffekten nach »verboten«, »erlaubt« und geboten katalogisiert. Obwohl Gesang damit schon viele Einwände gegen solche Eingriffe übergangen hat, formuliert er einige - wenn auch sehr weite - Grenzen.

Eine Grenze besteht in der Abweisung einer staatlich organisierten Eugenik ("zentralen Verbesserungen«, 46), da sie die Freiheit zu sehr einschränken würde. ${ }^{41}$ Dieses Szenario hält Gesang für unwahrscheinlich. An einer dezentralen, marktgesteuerten Eugenik hat Gesang weniger auszusetzen. ${ }^{42}$

Die zweite Grenze folgt aus der drohenden Ausweitung sozialer Ungleichheit bei dezentraler Verbesserung: Ungleichheit sei zwar als Phänomen unausrottbar, doch eine naturale Verfestigung sozialer Unterschiede, die einsetzen würde, wenn nur Reiche sich und ihre Kinder technisch "verbessern" könnten, wäre selbst durch harte Arbeit »nicht einholbar» (51). Daher vertritt Gesang eine staatliche Pflicht zur Finanzierung eines »kompensatorischen Enhancement«; einer Verbesserung von Menschen, die von der "natürlichen Lotterie» schlechter gestellt wurden $(69,71,151)$. Im Idealfall Gesangs wäre eine solche Behandlung kein Zwang, sondern nur eine Möglichkeit, seine Stellung auf dem Arbeitsmarkt $\mathrm{zu}$ verbessern (was allerdings schon heute zumindest für Arbeitslose staatliche Pflicht ist). Hier tritt eine Spannung zur Ablehnung zentraler Verbesserungen hervor; zwischen erster und zweiter Grenze knirscht es also.

Die dritte Grenze ist - trotz allem - wieder anthropologisch: Die veränderten Körper müssen zumindest noch als Menschen erkennbar sein $(75,136)$, und zwar als die Menschen, die sie vorher waren (78). Diese Grenze ist allerdings nur eine Auffangposition, da Gesang sowohl menschenrechtliche Skrupel (da die Natur an sich wertlos und das Prädikat "Mensch" fließend sei, 130) wie Einwände von professionellen Ethikern (als intellektuelle Sondermeinungen, 93, 119) ablehnt.

Am Ende steht eine breite Lizenz für eine technische Perfektionierung des Menschen. Sie sei nicht nur erlaubt, sondern zum Teil sogar geboten. Darin kann man einen Ausläufer der Spätromantik sehen: Zum einen suggeriert die Logik des »Idealfalls", die Technik sei der neutrale Motor einer blühenden Zukunft, ${ }^{43}$ zum anderen ist eine außerhalb von sozialen Bezü-

${ }^{41}$ Genauer geht es um die »Freiheit der Fortpflanzung« (46).

42 „Rein private Vorteile sind keine Problem für die soziale Ordnung« (63; zum Privaten auch Lecce, $239 \mathrm{ff}$.).

${ }^{43}$ Gesang hat kaum Skrupel: "So dürfe die Zahl der unglücklichen, antriebslosen und frustrierten Menschen auf der Welt ... abnehmen, wenn Mittel wie Prozac verstärkt eingesetzt werden. ... Das könnte auch die Kriminalität senken«(60). Dies erinnert an die Feier der Technik bei Walter Rathenau oder den Gebrüdern Jünger. 
gen gedachte »Selbstverwirklichung « zumindest ein wichtiges Motiv. ${ }^{44}$ Es ist also eine verfehlte Selbstverortung, wenn Gesang sich wiederholt gegen die Romantik wendet $(5,115,155)$, da sie den Fortschritt aufhalten wolle. Eher müsste er gegen die Weimarer »Klassik« polemisieren, denn vor allem sie artikulierte moralisch-perfektionistische Gedanken und die Vorstellung einer tiefen und reichen (und darum erhaltenswerten) Natur. Beides lehnt Gesang ab (93).

Zumindest einer der von Gesang Kritisierten ist tatsächlich ein Klassiker: Ein Mitglied des Presidents Council on Bioethics, den Gesang hier für seinen moralischen Perfektionismus kritisiert (»Er vertritt ein Ideal vom strue human flourishing «, 93), war Michael Sandel. Dieser hat seine Position 2007 in einem kleinen Büchlein verdeutlicht, das 2008 - erweitert um ein Vorwort von Jürgen Habermas - auf deutsch erschien. Darin überrascht Sandel mit einer recht liberalen Position: Er hat nichts gegen gentechnische Eingriffe per se einzuwenden (31) und befürwortet sogar eine Forschung mit embryonalen Stammzellen, solange - und damit sind wir schon beim Kern - die kommerzielle Nutzung eingeschränkt werde (148). Es geht ihm also nicht einfach um die ethische Bewertung einer Technik, sondern um eine Bewertung der sozialen Kontexte, in die sie eingebettet ist. Von ihnen hängt ab, ob wir eine Praxis erlauben sollten oder nicht; von ihnen zu abstrahieren heißt vom Problem zu abstrahieren.

Sandel argumentiert also realitätsnäher, was man schon daran merkt, dass sein Buch voller realer Beispiele ist (nicht ausgedachter "Herr Meier «-Beispiele wie bei Gesang, 142). Darin kommt er der eingangs besprochenen Yuracko nahe; sogar seine Herangehensweise ist ähnlich: Er geht davon aus, dass es ernstzunehmende moralische Intuitionen gibt (Habermas spricht von "moralischen Gefühlen«, 10), die uns bei der Einführung solcher Techniken vorsichtig machen sollten. Die Frage ist nur, wie sie philosophisch auszubuchstabieren sind. Es reicht für Sandel nicht, das herkömmliche liberale Vokabular von Autonomie und Fairness in Anschlag zu bringen, denn das würde auch von Befürwortern der Eugenik (wie Allen Buchanan u.a.) erfolgreich benutzt. ${ }^{45}$ Daher spricht Sandel - wie Yuracko - eher vom "Gedeihen «. ${ }^{46}$

Warum schadet genetische Optimierung aber dem menschlichen Gedeihen? Hier führt Sandel Argumente aus den oben genannten zwei Be-

44 "[S]olange eine Verbesserung zu mir und meinen anderen Wünschen passt und ich den Wunsch nach ihr bejahe ..., verwirkliche ich mich" (88). Eine solche Praxis könne nicht als »inauthentisch" kritisiert werden.

45 »Eine Ethik der Autonomie und der Gleichheit kann nicht erklären, was mit der Eugenik nicht stimmt» (101, vgl. 30, 94, 97). Gegen Habermas wendet Sandel ein, Autonomie sei kein starkes Argument gegen Klonen, da die Alternative - das Ausgeliefertsein an die Lotterie der Natur - ebensowenig autonom sei (29).

46 Sandel, 44. Da von Taylor schon die Rede war, sei bemerkt, dass auch Alasdair MacIntyre nun von "flourishing"spricht (Dependent Rational Animals. Why Human Beings Need the Virtues, London 1999). 
reichen an. Sein anthropologischer Punkt lautet, »ein Aspekt unseres Menschseins" (47) sei der "Sinn für das Geschenktsein« (107). Das scheint der von Habermas angeführten "Unverfügbarkeit des eigenen Anfangs" nahezukommen. ${ }^{47}$ Doch Sandel deutet die Auswirkung der Eugenik auf sie umgekehrt: Befürchtet Habermas eine Einschränkung der Freiheit, da es neue Abhängigkeiten gebe, beobachtet Sandel eher eine Ausweitung derselben (»Explosion, nicht ... Erosion der Verantwortung« 108). Die Fähigkeit, das natürliche Sein einer Person (einschließlich der eigenen) als gegeben hinnehmen zu können, sei die Grundlage für »Schlüsselelemente unserer moralischen Landschaft - Demut [humility, CH], Verantwortung und Solidarität« (107). Bei einer Ausweitung von Verfügungsmacht hingegen schwinde die alte "Gegenseitigkeit aufgrund von Ungewissheit« (111). Es geht Sandel also nicht nur um die moralischen Gefühle, die man hat, wenn man an solche Optimierungen denkt, sondern vor allem um die, die man nicht mehr hat, würden sie »routinemäßig praktiziert» (112). ${ }^{48}$

Die technische Perfektionierung berührt also auch den Bereich, der im moralischen Perfektionismus diskutiert wird: Sandel kritisiert ja nicht die technischen Details per se, sondern ihre Auswirkungen auf die menschlichen Entwicklungsmöglichkeiten. Um es in den obigen Termini auszudrücken: Diese Praxis wäre keineswegs "neutral«, denn (mit Wall gesagt) Verbot wie Erlaubnis hätten Auswirkungen auf die Menschen, und zwar auf Beteiligte wie auf Unbeteiligte. Es gibt (mit Yuracko gesagt) Dinge, die besser nicht zur Wahl stehen sollten, da schon das Haben einer solchen Wahl korrumpieren kann. ${ }^{49}$ Aus moralisch-perfektionistischer Sicht verschlechterte sich "der Mensch" durch eine solche Praxis, so wäre das Argument zu verlängern, nicht weil sich etwas innerhalb der moralischen Dimension veränderte, sondern weil diese selbst zurückginge - und mit ihr die Menschlichkeit des Menschen.

Der zweite Einwand Sandels ist ein sozialtheoretischer. Die Ausweitung der Verantwortlichkeit selbst noch auf unsere Gene stehe nicht im luftleeren Raum, sondern folge einer gesellschaftlichen Dynamik: Sie ist marktgetrieben. Dies nicht nur in dem Sinne, dass stets jemand daran verdient (etwa die "Pharmafirmen«, 35, mit Umsätzen von 1 Mrd. \$ nur im Ritalin-Markt, 80), sondern auch im Sinne des Habitus' der Konkurrenz. In der Ausweitung dieser "Ethik des Eifers und Trachtens" (50) sieht Sandel einen "ängstlichen Auswuchs der Beherrschung und Dominanz« (82), also - kurz gesagt - nichts Gutes.

${ }^{47}$ Jürgen Habermas: Die Zukunft der menschlichen Natur. Auf dem Weg zu einer liberalen Eugenik? Frankfurt/M. 2001, 29.

${ }_{48}$ Der "sense of giftedness" ist für Sandel übrigens nicht von der Religion abhängig (eine weitere Parallele zu Habermas). Das darin ausgedrückte Faktum, dass man sich nicht selbst gemacht hat, kann niemand pachten.

49 So habe ein Sportler Ärger von seinen Mitspielern bekommen, weil er sich nicht gedopt habe (Sandel, 110). 
Der Blick auf die abschreckenden Folgen, die bei Durchsetzung einer Praxis genetischer Optimierungen drohen, führt zunächst auf die Frage, in welcher Gesellschaft wir leben wollen - nach Sandel jedenfalls nicht in einer, "in der sich Eltern gezwungen sehen, ein Vermögen dafür auszugeben, völlig gesunde Kinder ein paar Zentimeter größer zu machen« (39). Dies führt Sandel konsequent weiter zu einer Kritik auch der nicht-genetischen Vorformen des "technischen" Optimierens, die für Gesang ein Argument für die Einführung genetischer Formen waren. Tatsächlich führt Sandel einen Reigen absurdester Beispiele auf, die zum Nachdenken veranlassen müssen. ${ }^{50}$ Einen größeren Anspruch hat dieses kleine Buch gar nicht, aber ihn hat es mehr als erreicht. Michael Sandel, der sein Projekt über die »moralischen Grenzen von Märkten« (19) soeben auf BBC 4 weiterführt, ${ }^{51}$ hat sich damit einmal mehr nicht nur als brillanter Autor, sondern auch als ein großer öffentlicher Intellektueller erwiesen.

Eine Einsicht, die man der Zusammenschau dieser im Grunde sehr verschiedenen Bücher entnehmen kann, ist die, dass vom Perfektionismus in ethischer und politischer Hinsicht in Zukunft noch einiges zu erwarten ist. Er wird aber nur dann weiterführende Resultate erzielen können, wenn er seinen Elfenbeinturm verlässt und sich stärker anthropologischen und sozialtheoretischen Forschungen öffnet. Wie insbesondere Marks, Sandel und Yuracko gezeigt haben, eröffnet das der Sozialphilosophie vielversprechende neue Wege - selbst wenn sie auf diesen Wegen einigen alten Bekannten begegnen dürfte.

\author{
Christoph Henning \\ Universität St. Gallen \\ Fachbereich für Philosophie \\ Gatterstrasse 1 \\ 9010 St. Gallen \\ Schweiz \\ christoph.henning@unisg.ch
}

50 Sandel nennt u.a. Abwehrspieler, die (intendiert) $192 \mathrm{~kg}$ wiegen (55); amerikanische Jugendsportverbände, die »elternfreie Zonen« einrichten müssen, um die Schüler vor dem Eifer ihrer Eltern zu schützen, sowie eine massive Zunahme von sportlichen »Ermüdungsverletzungen " (74); die enormen (und steigenden) Umsätze privatisierter "Prüfungsvorbereitung " und "Hochschulzugangsberater", die mit Slogans werben wie "ich lenke Leben" (76f.); sowie den Anstieg von Medikamenten wie Ritalin um 1700\% in 15 Jahren (80).

51 Wenn es erlaubt ist, sei dieser Link empfohlen: http://www.bbc.co.uk/programmes/ b00kt7rg. 